\title{
Colon cancer in a 12-year-old girl with hypertriglyceridemia
}

\author{
Monica Pedroni ${ }^{1}$, Maurizio Ponz de Leon ${ }^{1}$, Luca Reggiani Bonetti ${ }^{1}$, Alessandra Viel ${ }^{2}$, Davide Noto ${ }^{3}$, Fabio \\ Nascimbeni ${ }^{4}$, Paola Sena ${ }^{5}$, Luca Roncucci ${ }^{1,4}$ \\ 1Dipartimento di Scienze Mediche e Chirurgiche, Università di Modena e Reggio Emilia, Modena 41125, Italy. \\ 2Dipartimento di Ricerca Traslazionale e Oncologia Molecolare, Centro di Riferimento Oncologico, Aviano 33081, Italy. \\ ${ }^{3}$ Dipartimento Biomedico di Medicina Clinica e Specialistica, Università di Palermo, Palermo 90100, Italy. \\ ${ }^{4}$ Dipartimento di Medicina Interna, Generale, D'Urgenza e Post-Acuzie, Azienda Ospedaliero-Universitaria di Modena e Reggio \\ Emilia, Modena 41126, Italy. \\ ${ }^{5}$ Dipartimento Chirurgico, Medico, Odontoiatrico e di Scienze Morfologiche, Università di Modena e Reggio Emilia, Modena \\ 41125, Italy.
}

Correspondence to: Luca Roncucci, Dipartimento di Scienze Mediche e Chirurgiche, Università di Modena e Reggio Emilia, Medicina Metabolica, Ospedale Civile di Baggiovara, Via Giardini 1355, Modena 41126, Italy. E-mail: luca.roncucci@unimore.it

How to cite this article: Pedroni M, Ponz de Leon M, Reggiani Bonetti L, Viel A, Noto D, Nascimbeni F, Sena P, Roncucci L. Colon cancer in a 12-year-old girl with hypertriglyceridemia. Metab Target Organ Damage 2022;2:1.

https://dx.doi.org/10.20517/mtod.2021.12

Received: 27 Sep 2021 First Decision: 29 Nov 2021 Revised: 10 Dec 2021 Accepted: 22 Dec 2021 Published: 4 Jan 2022

Academic Editor: Sonia M. Najjar Copy Editor: Yue-Yue Zhang Production Editor: Yue-Yue Zhang

\begin{abstract}
Colorectal cancer is usually considered a disease of the elderly; however, in a small fraction of patients (2\%-3\% of all affected individuals), colorectal malignancies may develop earlier. The reasons whereby some individuals develop colorectal cancer at a young age are poorly understood. In a 12-year-old girl, a malignancy was diagnosed in the ascending colon. There was no familial history of Lynch syndrome or familial adenomatous polyposis. The metabolic profile of the patient revealed hypertriglyceridemia and low high-density lipoprotein cholesterol levels at nine years, then diagnosed as familial hypertriglyceridemia due to a constitutional mutation in the APOA5 gene (c. $427 \mathrm{deIC}$ ). Moreover, variants possibly increasing the risk of cancer were detected in MSH6 (c.3438+11_3438+14delCTTA, intron 5) and APC (11307K). The patient showed a rather unusual dietary pattern, since her basic alimentation from weaning consisted almost exclusively of meat homogenates and, subsequently, roasted meat or cutlets. Other foods, including fish, vegetables, sweets, and pasta, were refused. In this case, genetic and environmental factors could have acted in a particularly accelerated manner. Indeed, the genetic background of the patient (familial hypertriglyceridemia and polymorphisms predisposing to colorectal cancer) may have favored a dietary-driven colorectal carcinogenesis, resulting in an extremely early onset development of
\end{abstract}


malignancy.

Keywords: Colorectal cancer, hypertriglyceridemia, APOA5, MSH6, APC, case report

\section{INTRODUCTION}

Colorectal cancer is common in elderly individuals but rather infrequent under the age of 50 years, although recent reports show that the incidence is increasing in young people in many countries ${ }^{[1]}$; the disease may be considered a rarity in the first and second decades of life ${ }^{[2]}$. While approximately $3 \%$ of all colorectal malignancies show a genetic origin, the fraction raises to $20 \%-30 \%$ among people who develop the tumor before the age of 35-40 years ${ }^{[3,4]}$. It follows that in early or very early onset colorectal cancer a possible genetic background, in particular Lynch syndrome or familial adenomatous polyposis, should always be taken into consideration ${ }^{[5,6]}$. However, the large majority of colorectal neoplasms developing in young individuals are not clearly associated with hereditary factors - at least within the limits of our present knowledge - and their etiology remains undetermined.

We describe the unusual case of a 12-year-old girl in whom a Dukes C (T4N1Mo) carcinoma of the ascending colon developed in the absence of a documented genetic syndrome or a background of polyposis. Rather unexpectedly, the metabolic profile of the patient (familial hypertriglyceridemia with evidence of a known heterozygous frameshift mutation in the APOA5 gene), and possibly predisposing variants in the $M S H 6$ and $A P C$ genes, together with an unusual dietary pattern (excessive consumption of meat) may have been the basis of an advanced malignant tumor before adolescence.

\section{CASE REPORT}

The index case was born in 2004 with a caesarean delivery (weight at birth $3150 \mathrm{~g}$ ). Since then, physical and mental development appeared in the normal range; she entered school at age 5 and attended regular courses. At age 9, she was diagnosed with hypertriglyceridemia and low high-density lipoprotein (HDL) cholesterol levels [Table 1], which was treated irregularly with polyunsaturated fatty acids. The youngest sister, the father, and the paternal grandfather showed a similar lipid profile. At age 10, she underwent appendicectomy (2014).

In October 2015, she started to complain of fatigue, palpitations, and mild abdominal pain. After almost one year, blood tests revealed severe anemia $(\mathrm{Hb}: 6.0 \mathrm{~g} / \mathrm{dL}$ ), with low serum iron levels, and a fecal occult blood test was positive. In September 2016, at age 12 and 7 months, a colonoscopy showed a large and substenosing tumor of the ascending colon. A PET (positron emission tomography) scan revealed accumulation of fluorodeoxyglucose in the right colon and in two small pelvic nodules. Two cycles of chemotherapy (FOLFOXIRI, folinic acid, 5-fluorouracil, oxaliplatin, and irinotecan) were executed before surgery. The patient underwent a right hemicolectomy in November 2016. At anatomical examination, a substenosing lesion of $1.5 \mathrm{~cm}$ was observed in the right colon. Histologic diagnosis was poorly differentiated adenocarcinoma - with aspects of signet ring cell carcinoma - with tumor budding, neural and vascular invasion, and metastasis in 3 of 57 lymph nodes (T4N1Mx). Two 8-10 mm peritoneal nodules resected with the colon showed fibrotic tissue free of malignant cells. The patient recovered promptly and had her menarche two months after surgery; she underwent eight more cycles of chemotherapy (FOLFOX, folinic acid, 5-fluorouracil, and oxaliplatin) in 2017. In July 2017, the patient was in good clinical condition and well developed. Her height was $149 \mathrm{~cm}$ and weight was $48 \mathrm{~kg}$. She had a disease relapse in November 2017 (a $13 \mathrm{~cm}$ mass of the ovary and four peritoneal masses), so she underwent ovariectomy, peritonectomy, and intraperitoneal hyperthermic antiblastic perfusion with cisplatin and mitomycin. In November 2020, she 
Table 1. Serum lipid pattern over time of the four family members with hypertriglyceridemia

\begin{tabular}{|c|c|c|c|c|c|c|c|}
\hline & \multicolumn{7}{|c|}{ Calendar year } \\
\hline & 2007 & 2011 & 2012 & 2013 & 2014 & 2015 & 2017 \\
\hline \multicolumn{8}{|l|}{ Grandfather I-1 } \\
\hline Triglycerides (mg/dL) & 439 & & & & & & \\
\hline Total cholesterol (mg/dL) & 185 & & & & & & \\
\hline LDL cholesterol (mg/dL) & - & & & & & & \\
\hline $\mathrm{HDL}$ cholesterol (mg/dL) & 23 & & & & & & \\
\hline \multicolumn{8}{|l|}{ Father II-1 } \\
\hline Triglycerides (mg/dL) & 405 & 711 & & & & & 232 \\
\hline Total cholesterol (mg/dL) & 157 & 247 & & & & & 194 \\
\hline LDL cholesterol (mg/dL) & - & - & & & & & 134 \\
\hline HDL cholesterol (mg/dL) & 23 & 25 & & & & & 34 \\
\hline \multicolumn{8}{|l|}{ Patient (proband) III-1 } \\
\hline Triglycerides (mg/dL) & & & 114 & 198 & 333 & 179 & 332 \\
\hline Total cholesterol (mg/dL) & & & 205 & 195 & 208 & - & 241 \\
\hline LDL cholesterol (mg/dL) & & & 147 & - & 151 & - & 154 \\
\hline HDL cholesterol (mg/dL) & & & 33 & 24 & 31 & - & 38 \\
\hline \multicolumn{8}{|l|}{ Sister III-3 } \\
\hline Triglycerides (mg/dL) & & & 284 & & 123 & 214 & 66 \\
\hline Total cholesterol (mg/dL) & & & 169 & & 232 & 211 & 190 \\
\hline LDL cholesterol (mg/dL) & & & - & & 164 & 147 & 130 \\
\hline HDL cholesterol $(\mathrm{mg} / \mathrm{dL})$ & & & 19 & & 43 & - & 48 \\
\hline
\end{tabular}

had a new relapse near the colonic anastomosis and a suprapubic nodule in the rectious muscles of the abdomen, which were surgically removed in January 2021 , along with a new peritonectomy with intraperitoneal hyperthermic perfusion of cisplatin and mitomycin. Then, she underwent six more cycles of FOLFOXIRI (folinic acid, 5-fluorouracil, oxaliplatin, and irinotecan) plus bevacizumab. As of November 2021, she is in a stable condition, and she will be clinically re-evaluated in February 2022.

\section{Colorectal cancer molecular studies}

From the genealogic tree of the family [Figure 1], no pattern of vertical transmission of cancer was clearly evident, and the proband was the only case in the family. As a matter of fact, in rare cases, Lynch syndrome may initiate with a new mutation, especially in a young patient, with neoplasms of the proximal colon and histologic pattern of mucinous or signed ring cell carcinoma ${ }^{[7]}$. Moreover, by endoscopy and anatomical examination of the resected colon, no polyps or background of diffuse polyposis could be observed. Similarly, features of Peutz-Jeghers or Cowden disease ${ }^{[8,9]}$ were absent in the proband and in her first-degree relatives.

However, because of the unusual case, the possible genetic origin of the tumor was studied using next generation sequencing (NGS) techniques. In particular, a TruSeq Custom Amplicon workflow was applied in searching for constitutional sequence variants in genes which increase the risk of colorectal cancer. The panel included all exons and \pm 20 padding intronic regions of the following genes: $A P C, M U Y Y H, M L H 1$, MSH2, MSH6, PMS2, EPCAM, STK11, PTEN, SMAD4, and BMPR1A. Variants with global minor allele frequency of $<1 \%$ were confirmed by Sanger sequencing ${ }^{[10,11]}$. 


\section{I}

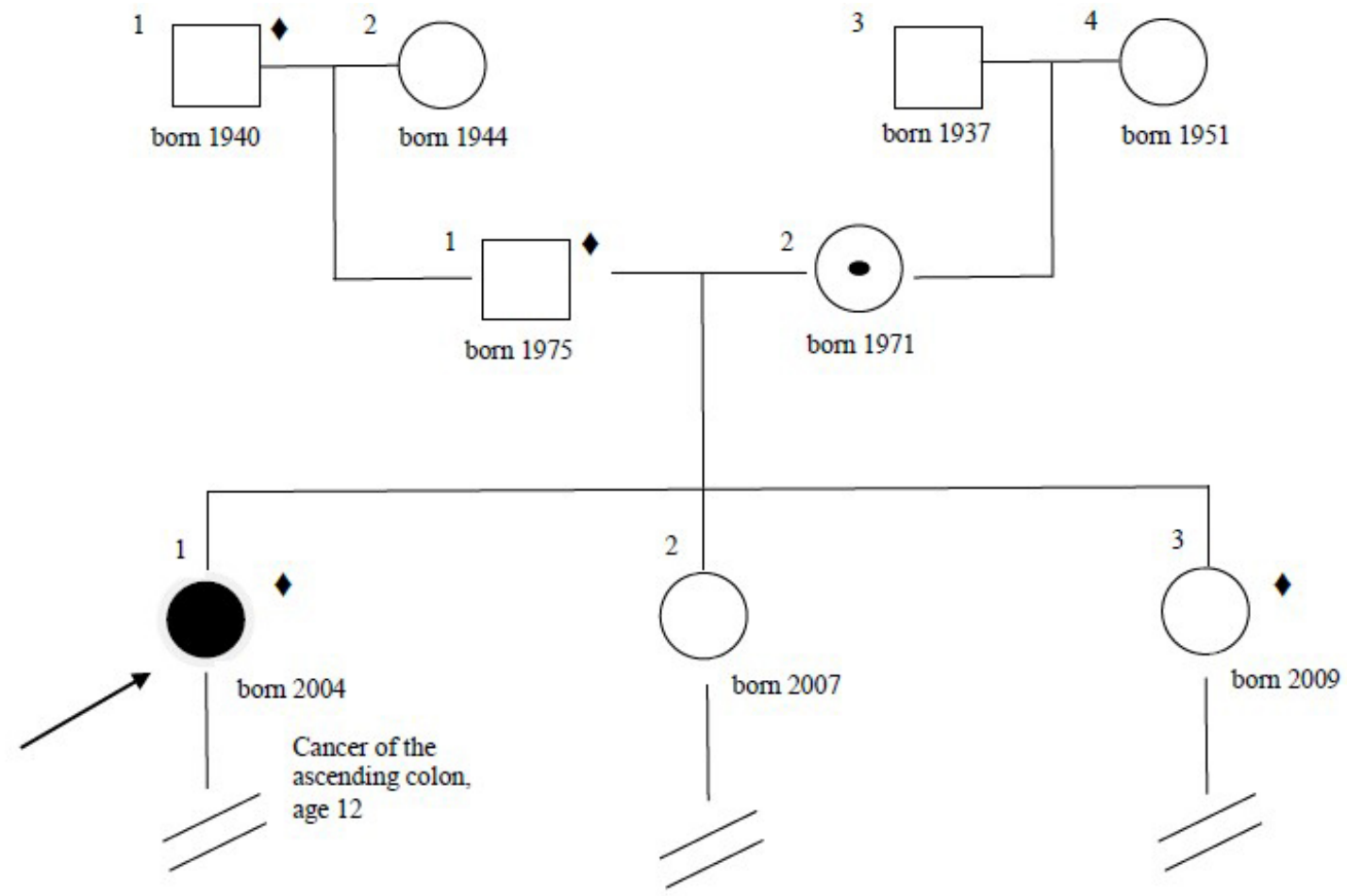

3

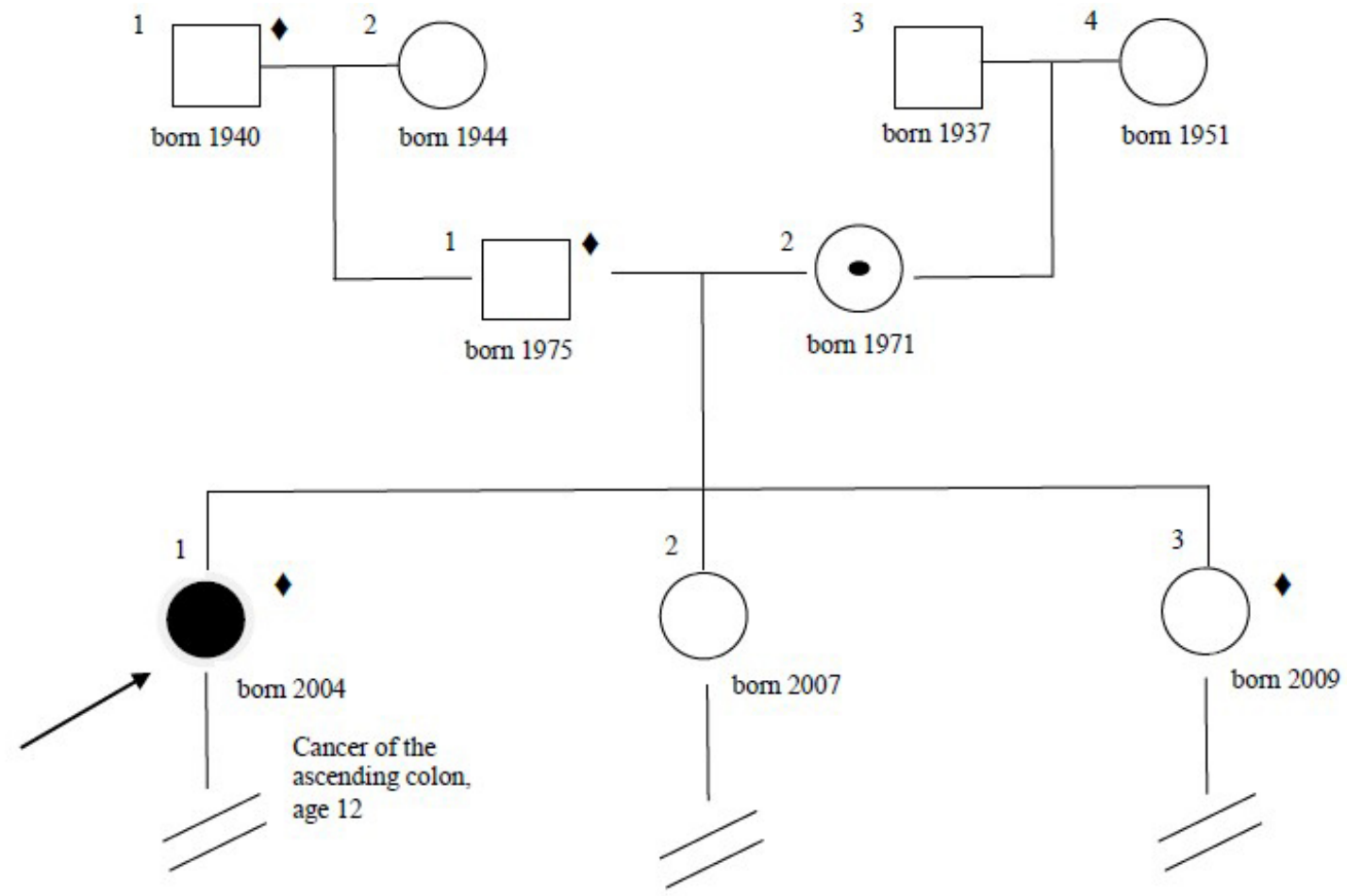

II

III

- The diamond beside the symbols indicates hypertriglyceridemia
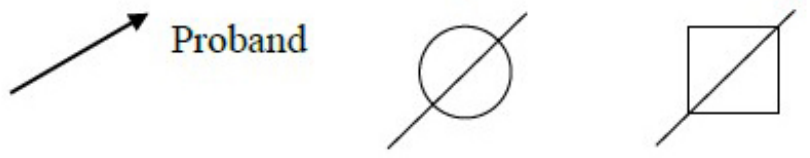

Deceased

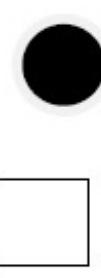

\section{Colon cancer}

Male<smiles>C1CCC2CCCCC2CC1</smiles>

Female

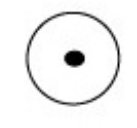

Myoma of the uterus

Figure 1. Genealogical tree of the family.

The NGS study revealed wild-type sequences and no large gene deletion/duplications. A four-base deletion at intron 5 in the MSH6 gene was detected (c.3438+11_3438+14delCTTA). This constitutional alteration is considered as a variant of uncertain significance, according to the international database (InSight Variant Database and Universal Mutation Database), not clearly associated with Lynch families or segregating in kindreds. Moreover, the missense variant c.3920 T>A (p.Ile1307Lys, also known as I1307K) was detected in the APC gene [Figure 2]; this is a low-penetrance, relatively frequent (especially in Ashkenazi Jews) mutation associated with an increased risk of colorectal cancer, but with no definite role in hereditary colorectal cancer and familial adenomatous polyposis ${ }^{[12,13]}$. In addition, loss of heterozygosity studies in the tumor tissue showed that the variant maintained its heterozygous state. The immunohistochemical evaluation of the mismatch repair proteins showed normal expression of the four main proteins (MLH1, $\mathrm{MSH} 2, \mathrm{MSH} 6$, and PMS2) in the resected tumor [Figure 3] ${ }^{[14]}$. 


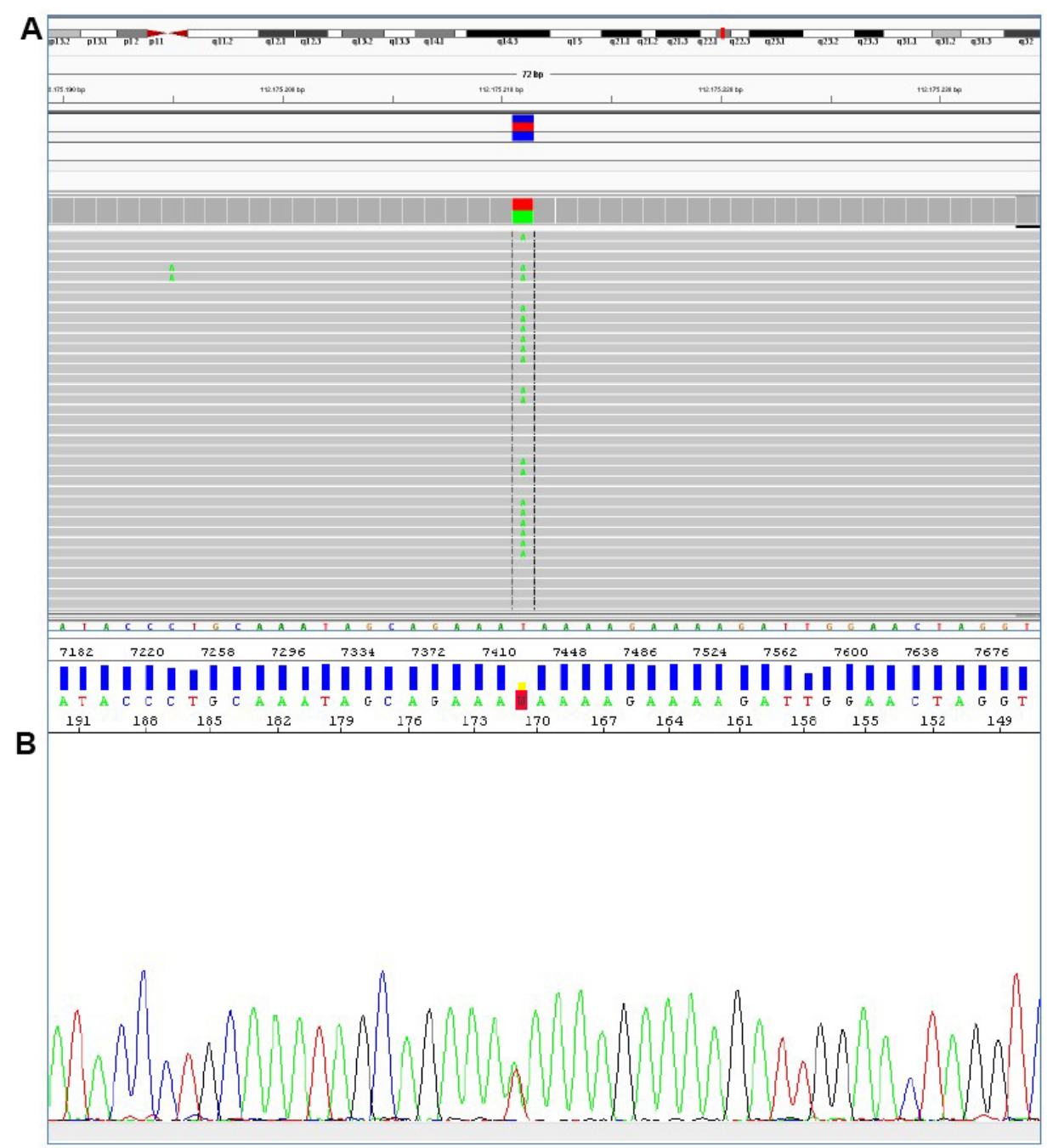

Figure 2. (A) The I1307K variant in the APC gene, by Next Generation Sequencing (NGS). (B) The same variant confirmed by Sanger sequencing.

It is unlikely that the two above-mentioned constitutional alterations may explain "in se" the development of cancer at age 12 in our proband. However, it is possible that these variants contribute to create a susceptible background which may interact with other genetic or exogenous factors in the induction (and progression) of the colorectal malignancy.

\section{Dietary pattern of the index case}

Although mental attitude and behavior of the proband (index case) were otherwise normal when compared with other girls of her age, her dietary habits were actually rather unusual. From birth to the sixth or seventh month she followed a milk alimentation with humanized artificial milk. Weaning was started in the sixth month; however, the mother reported that she could feed the proband only with meat homogenates, since she refused any other type of food. From one year of life, meat was the basic alimentation of the proband, usually $50 \mathrm{~g}$ (approximately) three times per day, every day; this amounts to about $1 \mathrm{~kg}$ of meat every week. Meat was predominantly beef or chicken, and it was cooked roasted or dipped in bread crumbs. With increasing age (3-5 years of life), ham and wurstel were also eaten, together with small portions of bread and yogurt, but 2-3 portions of meat every day remained the basic alimentation. Pasta and vegetables were 


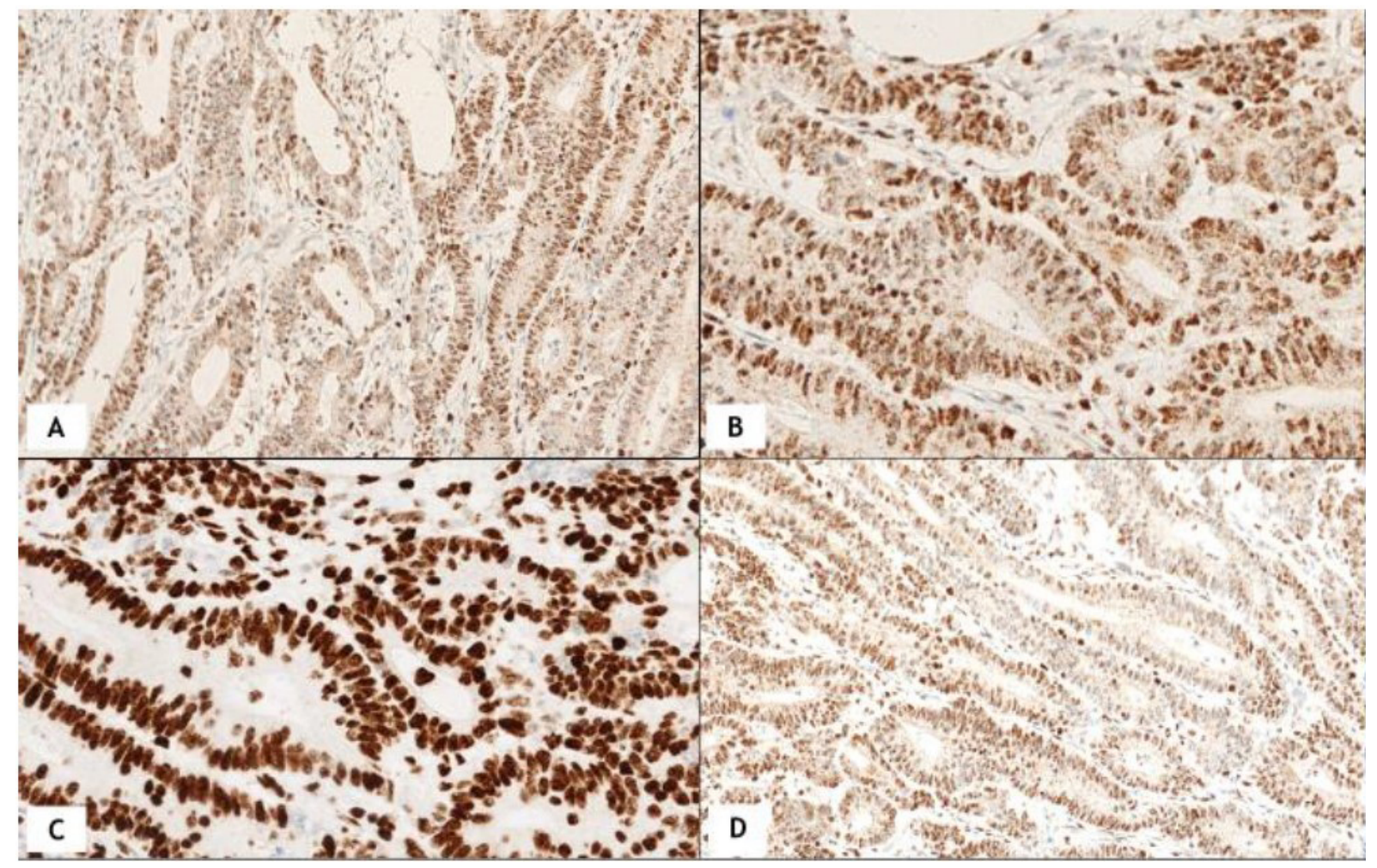

Figure 3. Typical immunohistochemical nuclear staining of the four mismatch-repair proteins in tumor cells of the carcinoma of the patient: MLH1 (A); PMS2 (B); MSH2 (C); and MSH6 (D). Magnification is 40x.

refused by the baby; when the mother insisted on forcing the proband to eat these foods, they were regularly vomited. Fish was never eaten because it was refused; similarly, at variance with other children, sweets and ice cream were not accepted. Meat was always cooked in the same way: roasted with some olive oil or dipped in bread crumbs, with some salt.

This meat-based dietary pattern was maintained up to the end of 2016 (and, thus, to the development of symptoms). In the last years, after the first surgical intervention, the continuous efforts of the mother and the suggestions of the medical personnel led to a change of the dietary habits, increasing the consumption of pasta and vegetables. The other members of the family, including those with high triglyceride levels, regularly consumed any kind of food, without restriction of dietary fat.

\section{Family history and lipid disorders}

The genealogical tree of the nuclear family is depicted in Figure 1. No other malignancy was reported among first-degree relatives and grandparents. The mother of the proband (II-2) underwent hysterectomy at age 38 because of myoma of the uterus. The father, born in 1975, had acute pancreatitis in 2011, at age 36; on that occasion, overweight-associated non-alcoholic fatty liver and hypertension were also detected. Four years earlier, in 2007, hypertriglyceridemia was diagnosed; blood tests were executed because of the known high lipid levels of his father (the paternal grandfather of the proband), who referred triglycerides values in the order of 500-1000 mg/dL. Hypertriglyceridemia was also diagnosed in the proband (age 9) and in one of her sisters (III-3 in the family tree, age 3). Thus, familial high triglyceride levels were present in four patients and in three consecutive generations. Moreover, a lifelong history of overweight was present in the father and in the younger sister of the proband. 
Table 1 summarizes the serum lipid levels that we could document in the four members of the family affected by hypertriglyceridemia. Fluctuations of values could depend on dietary recommendations and therapy (fibrates and/or fish oil). Total and LDL cholesterol were also moderately elevated in most samples, while HDL levels were constantly below the normal range.

NGS exome analysis using the Ion Torrent Platform ${ }^{[15]}$ revealed the presence of a heterozygous constitutional mutation in the APOA5 gene in the proband and her relatives (I-1, II-1, and III-3) affected by hypertriglyceridemia. The variant (c.427 delC) was a frameshift mutation causing a stop codon downstream and, thus, a truncated protein (p.Arg143Alafs $\left.{ }^{\star} 57\right)^{[16]}$. The deleterious APOA5 mutation was described previously by Evans et al ${ }^{[16]}$ and was associated with moderate-to-severe hypertriglyceridemia including in heterozygous carriers.

\section{DISCUSSION AND CONCLUSIONS}

We describe the case of a girl who had cancer of the large bowel very early in her life. Our initial hypothesis was that inheritance could play a determinant role in inducing the development of malignancy at 12 years of age; however, family history and molecular studies apparently excluded, or rendered extremely improbable, the role of genetics in this case. As a matter of fact, there was no family history of colorectal cancer or polyposis, immunohistochemistry of the main DNA mismatch repair proteins ( $M L H 1, M S H 2, M S H 6$, and $P M S 2)$ revealed a normal expression, and no truncating mutation in 11 cancer-related genes could be detected. The variants observed in MSH6 and APC, although possibly linked to an increased risk of tumors, were not associated with very early colorectal cancer occurrence, and they can hardly be invoked as the main factors for explaining the observed case.

We therefore turned our attention to hypertriglyceridemia, which ran in the family for at least three generations (including the proband), and to the peculiar alimentation of the index case, which consisted from birth almost exclusively of meat. High triglycerides levels and meat consumption have been associated with the development of colorectal cancer.

Evidence linking triglyceride levels to tumors in general is rather consistent. McKeown-Eyssen ${ }^{[17]}$ gathered epidemiological evidence that hypertriglyceridemia was associated with the risk of colorectal cancer. Among possible explanations, the author suggested an association of triglycerides with fecal bile acids (which promote colorectal carcinogenesis), increased insulin levels, and the fact that triglycerides might be indicators of energy available through the circulation for tumor cells. Other studies reported that high serum triglycerides levels were associated with an increased risk of colonic or colorectal adenomas ${ }^{[18-20]}$. In a large group of patients with prostate cancer, Hayashi et al. ${ }^{[21]}$ reported a correlation between triglyceride levels and incidence of the disease, especially in patients over the age of 60 years, and concluded that individuals with high triglyceride levels may be more vulnerable to prostate cancer. Among possible explanations. the authors suggested insulin resistance - which is closely associated with obesity and high lipid levels - inflammatory state, and oxidative stress. Similar results were reported in a recent study by Allott et al. ${ }^{[22]}$, in which the authors evaluated recurrence of prostate cancer after surgery. In a large study including 31,000 women recruited between 1995 and 1997, Lindemann et al. ${ }^{[23]}$ found a significant positive association between serum triglyceride levels with risk of endometrial cancer; the authors suggested that some of the effects related to triglycerides could be mediated by body mass. Since adipose tissue is a source of endogenous estrogens, unopposed estrogen may play a relevant role in the occurrence of endometrial cancer. Moreover, a recent meta-analysis reported that serum triglycerides levels are higher in premenopausal women with breast cancer ${ }^{[2]}$. To summarize, high triglyceride levels are closely associated with obesity and insulin resistance, which in turn induce a proinflammatory state that favors cell 
replication, activation of mutagens (products of oxidative stress), and ultimately cancer ${ }^{[25]}$. In accordance with this hypothesis and sequence of events, Otani et al. ${ }^{[26]}$ found that plasma levels of C-reactive protein - a marker of inflammation - were associated with a subsequent risk of colorectal cancer.

In our study, the proband and three of the relatives affected by hypertriglyceridemia were heterozygous carriers of a frameshift mutation in the APOA5 gene, which is associated with moderately to severely increased triglycerides levels ${ }^{[16]}$. Indeed, several genetic studies have shown a correlation between APOA5 variants and triglycerides ${ }^{[27,28]}$. In addition, APOA5 was associated with insulin resistance and metabolic syndrome ${ }^{[22]}$, two conditions whose association with systemic inflammation - potentially leading to cardiovascular diseases and neoplasm - has been documented.

The ingestion of meat has been associated with colorectal cancer in epidemiological studies; however, the strength of the association and types of meat involved were not always consistent. Studies on red meat consumption and tumors of the large bowel showed odd ratios in the order of $1.2-1.3^{[30,31]}$. Cotterchio et al. ${ }^{[32]}$, however, reported odd ratios of 1.4-1.7 and suggested that the cancer risk may be further elevated in individuals with some variants (CYP1B1 and SULT141) of the enzymes involved in carcinogen metabolism. Moreover, Ferrucci et al. ${ }^{[33]}$ found that red meat and pan-fried meat were associated with increased risks (1.7-2.0) of colorectal adenoma. In addition, Pan et al.$^{[34]}$ found evidence that consumption of red meat was significantly linked with an increased risk of total, cardiovascular, and especially cancerrelated mortality.

The reasons whereby meat consumption increases the risk of some types of cancer, in particular large bowel cancer, remain unclear, at the point that Forman ${ }^{[35]}$, in 1999, entitled his editorial on this topic as "Meat and cancer: a relation in search of mechanisms". Among the hypothetical mechanisms which might be invoked, we can mention: (1) the risk due to the carcinogenic polycyclic aromatic hydrocarbons produced when meat is cooked at high temperature ${ }^{[36]}$; (2) the fact that cooked, pan-fried, salted, and processed meat might induce the formation of carcinogenic substances such as heterocyclic amines and other nitroso compounds $^{[37]}$; (3) a meat-based alimentation may induce changes of the intestinal bacterial flora, as well as the (possible) formation of bacterial metabolites with a known carcinogenic effect ${ }^{[38]}$; and (4) iron and especially the heme iron present in red meat might promote colorectal cancer through the ability of iron to gain and lose electrons and, thus, to participate to potentially deleterious free radical-generating reactions ${ }^{[39,40]}$.

In conclusion, it is likely that both triglycerides and meat consumption play some role in colorectal cancer development, although controversies do exist in many investigations and reproducibility of results has been rather poor. If we turn our attention to the young girl described in the report, we can imagine that two common putative etiological factors - triglycerides and meat - which are usually diluted with many other factors in the general population in this specific case could exert their oncologic effect to the maximum level, starting from the birth - or very early childhood - and continuing for 12 consecutive years. In addition, the genetic variants detected in $\mathrm{MSH} 6$ and APC colorectal cancer-associated genes might have rendered the patient more susceptible to cancer development.

This case emphasizes the possible role of metabolic factors and unbalanced diets in colorectal cancer occurrence. However, other factors and agents might have been involved or facilitated the role of triglycerides and meat, such as common polymorphisms associated with colorectal cancer risk ${ }^{[4]}$ or mutation of unknown (or unexplored) colorectal cancer-associated genes ${ }^{[42]}$. 


\section{DECLARATIONS}

\section{Acknowledgement}

The authors wish to thank Dr. Franco Berrino (Istituto Nazionale per lo studio e la cura dei Tumori, Milan, Italy) for helpful discussions. We wish to thank also the Associazione Ricerca Tumori Intestinali (ARTI, Modena, Italy) for support.

\section{Authors' contributions}

Made a substantial contribution to acquisition of clinical data, analysis, drafting and review of the manuscript: Ponz de Leon M, Noto D, Roncucci L

Made a substantial contribution to genetic and clinical chemistry analyses, drafting, and review of the manuscript: Pedroni M, Viel A, Nascimbeni F, Sena P

Made a substantial contribution to pathological analyses: Reggiani Bonetti L

All authors have read and approved the final version of the manuscript.

\section{Availability of data and materials}

Data sharing is not applicable to this article as no datasets were generated or analysed during the current study. Personal, genetic, and clinical data cannot be disclosed, of course; however further de-identified information may be requested to the corresponding author.

\section{Financial support and sponsorship}

None.

\section{Conflicts of interest}

All authors declared that there are no conflicts of interest.

\section{Ethics approval and consent to participate}

Written informed consent to undergo genetic testing, and to participate in this study was obtained from the parents of the child (under 16) described in this study.

\section{Consent for publication}

Although no personally identifiable information is reported in this study, informed consent for publication was obtained from the parents of the child (under 16) reported in this study.

\section{Copyright}

(C) The Author(s) 2022.

\section{REFERENCES}

1. Li N, Lu B, Luo C, et al. Incidence, mortality, survival, risk factor and screening of colorectal cancer: a comparison among China, Europe, and northern America. Cancer Lett 2021;522:255-68. DOI PubMed

2. Ganapathi S, Kumar D, Katsoulas N, et al. Colorectal cancer in the young: trends, characteristics and outcome. Int J Colorectal Dis 2011;26:927-34. DOI PubMed

3. Meyer JE, Narang T, Schnoll-Sussman FH, Pochapin MB, Christos PJ, Sherr DL. Increasing incidence of rectal cancer in patients aged younger than 40 years: an analysis of the surveillance, epidemiology, and end results database. Cancer 2010;116:4354-9. DOI PubMed PMC

4. Domati F, Maffei S, Kaleci S, et al. Incidence, clinical features and possible etiology of early onset ( $\leq 40$ years) colorectal neoplasms. Intern Emerg Med 2014;9:623-31. DOI PubMed

5. Giardiello FM, Allen JI, Axilbund JE, et al; US Multi-Society Task Force on Colorectal Cancer. Guidelines on genetic evaluation and management of Lynch syndrome: a consensus statement by the US Multi-Society Task Force on colorectal cancer. Gastroenterology 2014;147:502-26. DOI PubMed

6. Shussman N, Wexner SD. Colorectal polyps and polyposis syndromes. Gastroenterol Rep (Oxf) 2014;2:1-15. DOI PubMed PMC

7. Lynch HT, Shaw TG. Practical genetics of colorectal cancer. Chin Clin Oncol 2013;2:12. DOI PubMed

8. Resta N, Pierannunzio D, Lenato GM, et al; AIFEG. Cancer risk associated with STK11/LKB1 germline mutations in Peutz-Jeghers syndrome patients: results of an Italian multicenter study. Dig Liver Dis 2013;45:606-11. DOI PubMed 
9. Hansen-Kiss E, Beinkampen S, Adler B, et al. A retrospective chart review of the features of PTEN hamartoma tumour syndrome in children. J Med Genet 2017;54:471-8. DOI PubMed

10. Kamps R, Brandão RD, Bosch BJ, et al. Next-generation sequencing in oncology: genetic diagnosis, risk prediction and cancer classification. Int J Mol Sci 2017;18:308. DOI PubMed PMC

11. Pearlman R, Frankel WL, Swanson B, et al; Ohio Colorectal Cancer Prevention Initiative Study Group. Prevalence and spectrum of germline cancer susceptibility gene mutations among patients with early-onset colorectal cancer. JAMA Oncol 2017;3:464-71. DOI PubMed PMC

12. Laken SJ, Petersen GM, Gruber SB, et al. Familial colorectal cancer in Ashkenazim due to a hypermutable tract in APC. Nat Genet 1997;17:79-83. DOI PubMed

13. Houlston RS, Tomlinson IP. Polymorphisms and colorectal tumor risk. Gastroenterology 2001;121:282-301. DOI PubMed

14. Sukhokanjanachusak K, Pongpaibul A, Nimmannit A, Akewanlop C, Korphaisarn K. Clinicopathological characteristics and outcome of adolescent and young adult-onset microsatellite stable colorectal cancer patients. J Adolesc Young Adult Oncol 2021;10:573-80. DOI PubMed

15. Quail MA, Smith M, Coupland P, et al. A tale of three next generation sequencing platforms: comparison of Ion torrent, pacific biosciences and Illumina MiSeq sequencers. BMC Genomics 2012;13:341. DOI PubMed PMC

16. Evans D, Aberle J, Beil FU. Resequencing the apolipoprotein A5 (APOA5) gene in patients with various forms of hypertriglyceridemia. Atherosclerosis 2011;219:715-20. DOI PubMed

17. McKeown-Eyssen G. Epidemiology of colorectal cancer revisited: are serum triglycerides and/or plasma glucose associated with risk? Cancer Epidemiol Biomarkers Prev 1994;3:687-95. PubMed

18. Otani T, Iwasaki M, Ikeda S, et al. Serum triglycerides and colorectal adenoma in a case-control study among cancer screening examinees (Japan). Cancer Causes Control 2006;17:1245-52. DOI PubMed

19. Tabuchi M, Kitayama J, Nagawa H. Hypertriglyceridemia is positively correlated with the development of colorectal tubular adenoma in Japanese men. World J Gastroenterol 2006;12:1261-4. DOI PubMed PMC

20. Xie C, Wen P, Su J, et al. Elevated serum triglyceride and low-density lipoprotein cholesterol promotes the formation of colorectal polyps. BMC Gastroenterol 2019;19:195. DOI PubMed PMC

21. Hayashi N, Matsushima M, Yamamoto T, Sasaki H, Takahashi H, Egawa S. The impact of hypertriglyceridemia on prostate cancer development in patients aged $\geq 60$ years. BJU Int 2012;109:515-9. DOI PubMed

22. Allott EH, Howard LE, Aronson WJ, et al. Racial differences in the association between preoperative serum cholesterol and prostate cancer recurrence: results from the SEARCH database. Cancer Epidemiol Biomarkers Prev 2016;25:547-54. DOI PubMed PMC

23. Lindemann K, Vatten LJ, Ellstrøm-Engh M, Eskild A. Serum lipids and endometrial cancer risk: results from the HUNT-II study. Int $J$ Cancer 2009;124:2938-41. DOI PubMed

24. Wu J, Lei X, Pan X, Zeng X, Li W. Association between serum lipids and breast cancer risk in premenopausal women: systematic review and meta-analysis. J Int Med Res 2021;49:3000605211061033. DOI PubMed PMC

25. Chandler PD, Song Y, Lin J, et al. Lipid biomarkers and long-term risk of cancer in the Women's Health Study. Am J Clin Nutr 2016;103:1397-407. DOI PubMed PMC

26. Otani T, Iwasaki M, Sasazuki S, Inoue M, Tsugane S; Japan Public Health Center-Based Prospective Study Group. Plasma C-reactive protein and risk of colorectal cancer in a nested case-control study: Japan Public Health Center-based prospective study. Cancer Epidemiol Biomarkers Prev 2006;15:690-5. DOI PubMed

27. Hubacek JA. Apolipoprotein A5 and triglyceridemia. Focus on the effects of the common variants. Clin Chem Lab Med 2005;43:897902. DOI PubMed

28. Wang J, Ban MR, Kennedy BA, et al. APOA5 genetic variants are markers for classic hyperlipoproteinemia phenotypes and hypertriglyceridemia. Nat Clin Pract Cardiovasc Med 2008;5:730-7. DOI PubMed

29. Zheng XY, Zhao SP, Yan H. The role of apolipoprotein A5 in obesity and the metabolic syndrome. Biol Rev Camb Philos Soc 2013;88:490-98. DOI PubMed

30. Norat T, Bingham S, Ferrari P, et al. Meat, fish, and colorectal cancer risk: the European Prospective Investigation into cancer and nutrition. J Natl Cancer Inst 2005;97:906-16. DOI PubMed PMC

31. Sinha R, Peters U, Cross AJ, et al. Meat, meat cooking methods and preservation, and risk for colorectal adenoma. Cancer Res 2005;65:8034-41. DOI PubMed

32. Cotterchio M, Boucher BA, Manno M, Gallinger S, Okey AB, Harper PA. Red meat intake, doneness, polymorphisms in genes that encode carcinogen-metabolizing enzymes, and colorectal cancer risk. Cancer Epidemiol Biomarkers Prev 2008;17:3098-107. DOI PubMed PMC

33. Ferrucci LM, Sinha R, Graubard BI, et al. Dietary meat intake in relation to colorectal adenoma in asymptomatic women. Am J Gastroenterol 2009;104:1231-40. DOI PubMed PMC

34. Pan A, Sun Q, Bernstein AM, et al. Red meat consumption and mortality: results from 2 prospective cohort studies. Arch Intern Med 2012;172:555-63. DOI PubMed PMC

35. Forman D. Meat and cancer:a relation in search of a mechanism. Lancet 1999;353:686-7. DOI PubMed

36. Mosley D, Su T, Murff HJ, et al. Meat intake, meat cooking methods, and meat-derived mutagen exposure and risk of sessile serrated lesions. Am J Clin Nutr 2020;11:1244-51. DOI PubMed PMC

37. Bingham SA, Pignatelli B, Pollock JR, et al. Does increased endogenous formation of N-nitroso compounds in the human colon explain the association between red meat and colon cancer? Carcinogenesis 1996;17:515-23. DOI PubMed

38. Castellarin M, Warren RL, Freeman JD, et al. Fusobacterium nucleatum infection is prevalent in human colorectal carcinoma. Genome 
Res 2012;22:299-306. DOI PubMed PMC

39. Kossenas K, Constantinou C. Epidemiology, molecular mechanisms, and clinical trials: an update on research on the association between red meat consumption and colorectal cancer. Curr Nutr Rep 2021. DOI PubMed

40. Bastide NM, Chenni F, Audebert M, et al. A central role for heme iron in colon carcinogenesis associated with red meat intake. Cancer Res 2015;75:870-9. DOI PubMed

41. Zeng C, Matsuda K, Jia WH, et al; Genetics and Epidemiology of Colorectal Cancer Consortium (GECCO), Colorectal Transdisciplinary (CORECT) Study, Colon Cancer Family Registry (CCFR). Identification of susceptibility loci and genes for colorectal cancer risk. Gastroenterology 2016;150:1633-45. DOI PubMed PMC

42. Yurgelun MB, Boland CR. "New" cancer genes and inherited colorectal cancer risk: caveat emptor. Gastroenterology 2017;152:12-3. DOI PubMed 